\title{
CERTAIN PROBLEMS RELATED TO THE CUTTING OF A SIMPLY CONNECTED PLANE REGION BY A CONTINUUM*
}

\author{
BY \\ WALLACE ALVIN WILSON
}

1. If $R$ is a simply connected plane region whose frontier $F$ is bounded and $C$ is a simple arc lying in $R$ save for its end points, it is well known that $C$ enjoys the following properties:

(a) $R-R \cdot C$ consists of two simply connected regions $R_{1}$ and $R_{2}$;

(b) $C$ is a part of the frontiers of both $R_{1}$ and $R_{2}$;

(c) $F+C$ is the union of the frontiers of $R_{1}$ and $R_{2}$.

Nothing could be simpler than this theorem, but when one desires to use it, certain questions arise. For instance, what precisely are the frontiers of $R_{1}$ and $R_{2}$ ? If $F$ is the union of two continua $H$ and $K$, can $C$ be so drawn that its ends lie on two components of $H \cdot K$ ? If so, will the frontiers of the two regions be $C+H$ and $C+K$ ? These questions are readily answered in the case that $F$ is a simple closed curve, but the answers in this case do not always remain correct when $F$ is more complicated. In particular, a negative answer to the second question suggests the substitution of a more general continuum for $C$ and this in turn leads to a re-examination of the truth of the resulting extension of the theorem.

Such an extension has been made elsewhere $\dagger$ and it is intended in the present article to examine further these questions and certain related topics. The first and third of the questions suggested above are discussed in \$§3-6. The remainder of the paper deals with the situation arising when the arc $C$ is replaced by a special kind of irreducible continuum and with related questions of accessibility. In particular, it is shown that under certain broad conditions the second and third of the above questions can be answered in the affirmative if the $\operatorname{arc} C$ is replaced by a slightly more general continuum.

2. Notation. In addition to the common notation of the aggregate theory, the following will be used.

The whole plane is denoted throughout by $Z$. If $F$ is a continuum, a component of $Z-F$ whose frontier is $F$ is called a principal component; a component of $Z-F$ whose frontier is a proper part of $F$ is called secondary. A

* Presented to the Society, October 27, 1928; received by the editors August 20, 1928.

$\dagger$ W. A. Wilson, On irreducible cross-cuts of plane simply connected regions, American Journal of Mathematics, vol. 51, pp. 19-30. 
frontier set whose complement has at least two principal components is called regular.

The statement that $C$ is a continuum irreducible between the sets $A$ and $B$ does not imply that $C$ contains the whole of either $A$ or $B$.

The symbol $V_{\delta}(a)$ denotes the set of points whose distance from the point $a$ is less than $\delta$.

If $m$ and $n$ are two points (or point sets) in the plane separated by the closed set $C$, we say that $C$ is an $S(m, n)$. If $C$ is an $S(m, n)$, but no proper closed subset of $C$ is an $S(m, n)$, we say that $C$ is an irreducible $S(m, n)$.

3. We shall begin with the question of the nature of the frontiers of the regions $R_{1}$ and $R_{2}$ referred to in $\$ 1$. It is clear that this depends upon the degree of complexity of $F$ and that a satisfactory solution is scarcely possible unless restrictions are imposed on $F$. In a discussion of this kind the principal property of a simple closed curve is that it is the common frontier of its complementary domains; hence the natural generalization of such a curve is the regular frontier. The principal properties of such frontiers which will be used have been given by $\mathrm{C}$. Kuratowski* and the author. $\dagger$ The following theorem is also convenient.

THEOREM. Let $F$ be the frontier of a simply connected region $R$ and let $S$ be any other component of $Z-F$. If the frontier of $S$ is bounded, it is regular.

Let $f$ be the frontier of $S$ and let $m$ and $n$ be points of $R$ and $S$, respectively. As $f \subset F, f$ is an irreducible $S(m, n)$. Then $f$ is a regular frontier (see Kuratowski, loc. cit., p. 133).

4. THEOREM. Let $R$ be a simply connected region whose frontier $F$ is bounded and regular. Let $C$ be a simple arc joining the points $a$ and $b$ of $F$ and contained in $R+a+b$. Let $R \cdot C$ divide $R$ into $R_{1}$ and $R_{2}$ with frontiers $F_{1}$ and $F_{2}$, respectively. Then $F$ contains either 0,1 , or 2 proper sub-continua irreducible between $a$ and $b$. In the first case $F_{1}=F_{2}=C+F$; in the second case $F_{2}=C+F$, $F_{1}=C+f_{1}$, where $f_{1} \subset F$ and is irreducible between $a$ and $b$; in the third case $F_{1}=C+f_{1}$ and $F_{2}=C+f_{2}$, where $f_{1}$ and $f_{2}$ are proper sub-continua of $F$ irreducible between $a$ and $b$.

If $F$ is irreducible between $a$ and $b, C+F$ has two principal complementary domains by Rosenthal's theorem. $\ddagger$ These obviously coincide with $R_{\mathbf{1}}$ and $R_{\mathbf{2}}$.

* C. Kuratowski, Sur les coupures irréductibles du plan, Fundamenta Mathematicae, vol. 6, pp. 130-145.

$\dagger$ W. A. Wilson, On bounded regular frontiers in the plane, Bulletin of the American Mathematical Society, vol. 34, pp. 81-90.

$\ddagger$ A. Rosenthal, Teilung der Ebene durch irreduzibele Kontinua, Sitzungsberichte der Münchener Akademie, 1919, p. 106. 
If $f_{1}$ is a proper sub-continuum of $F$ irreducible between $a$ and $b$, let $S_{1}$ and $S_{2}$ be the principal complementary domains of $C+f_{1}$. Since $F$ is regular, $M=F-f_{1}$ is connected and lies in one of these, say in $S_{2}$. Then $F \cdot S_{1}=0$ and $S_{1} \subset R$. It readily follows that $S_{1}=R_{1}$ or $S_{1}=R_{2}$, say the former. Since $F_{1}+F_{2}=C+F$, this shows that $F_{2} \supset M$. Let $N=F \cdot F_{2}$. Let $m$ be a point of $R_{2}$ and $n$ a point of some principal component of $Z-F$ other than $R$. Since $F$ is regular, $N$ is not an $S(m, n)$ unless $N=F$. Then $F_{2}=C+F$ and a repetition of the first part of this paragraph shows that another proper subcontinuum of $F$ irreducible between $a$ and $b$ is impossible, when $N=F$.

If $N \neq F, N$ does not contain $f_{1}$ since $N \supset M$. As neither $N$ nor $C$ is an $S(m, n), N \cdot C$ is disconnected by Janiszewski's theorem* and $N$ contains $a$ and $b$. For the same reason $N$ contains a sub-continuum $f_{2}$ irreducible between $a$ and $b$. As $f_{1} \neq f_{2}$, the reasoning of the previous paragraph shows that $F_{2}=C+f_{2}$.

Finally, the existence of a third proper sub-continuum of $F$ irreducible between $a$ and $b$ is impossible by virtue of the second paragraph of the proof, as $C$ divides $R$ into but two regions.

5. A consequence of the theorem of $\$ 3$ is that $\$ 4$ applies to any simply connected plane region whose frontier is bounded and a part of the frontier of some other region.

It is natural to expect that, if the frontier $F$ of $R$ is regular, then the frontiers of the regions $R_{1}$ and $R_{2}$ into which $C$ divides $R$ are regular. The second case of the above theorem shows that this is not true. For, if $m, n$, and $p$ are points of $R_{2}, R_{1}$, and $Z-\bar{R}$, respectively, the frontier of $R_{2}$, which is $F+C$, is neither an irreducible $S(m, n)$ nor an irreducible $S(m, p)$.

A further consequence of the above theorem is that, if $a$ and $b$ are two points of a bounded regular frontier $F$ accessible by a simple arc from one of the components of $Z-F$, there are at most two sub-continua of $F$ irreducible between $a$ and $b$. This need not be true if either $a$ or $b$ is not arcwise accessible.

To determine precisely which of the three cases mentioned in the theorem applies, we need to know the nature of $F$ and the location of $a$ and $b$ on $F$. The various possibilities may be classified briefly as follows. The notation is that of $\$ 4$.

(I) $F$ is indecomposable.

* Z. Janiszewski, Sur les coupures du plan faites par des continus, Prace Matematyczno-Fizyczne, vol. 26, Theorem A. If $P$ and $Q$ are bounded closed sets, if $P \cdot Q$ is connected, and neither $P$ nor $Q$ is an $S(m, n)$, then $P+Q$ is not an $S(m, n)$. See also $S$. Strasziewicz, Fundamenta Mathematicae, vol. 4, p. 129. 
(a) If $a$ and $b$ are not on the same constituent of $F, F_{1}=F_{2}=C+F$.

(b) If $a$ and $b$ are on the same constituent of $F, F_{1}=C+f_{1}$ and $F_{2}=C+F$.

Examples of both these cases are easily constructed by using the methods of $\mathrm{K}$. Yoneyama†.

(II) $F$ is the union of two indecomposable continua. In this case $F$ is the union of two continua $H$ and $K, H \cdot K=\sum_{1}^{n} \alpha_{i}$, where $n \geqq 2$ and depends upon the number of principal components of $Z-F$, each $\alpha_{i}$ is a closed set, and both $H$ and $K$ are irreducible between each pair of sets $\left\{\alpha_{i}\right\}$. $\ddagger$ The various possibilities are as follows.

(a) If $a$ and $b$ are on different sets $\alpha_{i}, F_{1}=C+H$ and $F_{2}=C+K$. (For an example see Ex. 2 of Wilson, loc. cit. II, taking $C=c d$.)

(b) If $a$ and $b$ are on the same component of some $\alpha_{i}, F_{1}=C+F$ and $F_{2}=C+f$, where $f \subset \alpha_{i}$.

(c) If $a$ and $b$ are on the same set $\alpha_{i}$, but on different components, both $B$ and $K$ are irreducible between them, $F_{1}=C+H$, and $F_{2}=C+K$.

For we then have two sub-continua $f_{1}$ and $f_{2}$ of $F$ irreducible between $a$ and $b, F_{1}=C+f_{1}$, and $F_{2}=C+f_{2}$, which gives the contradiction $F_{1}+F_{2} \neq C+F$ unless $f_{1}=H$ and $f_{2}=K$. As a corollary it follows that, if two components of any $\alpha_{i}$ are arcwise accessible from $R$, both $H$ and $K$ are irreducible between them.

(d) If $a$ is on some $\alpha_{i}$ and $b$ on no $\alpha_{i}$, there are three possibilities. If $H$ is reducible between $a$ and $b, F_{1}=C+f_{1}$, where $f_{1} \subset H$, and $F_{2}=C+F$. If $H$ is irreducible between $a$ and $b$, but reducible between $b$ and some $\alpha_{j}$, $F_{1}=C+H$ and $F_{2}=C+K+f_{1}$, where $f_{1} \subset H$. If $H$ is irreducible between $b$ and every $\alpha_{i}, F_{1}=C+H$ and $F_{2}=C+F$.

(e) If neither $a$ nor $b$ is on any $\alpha_{i}$, we may have any of the three cases in $\$ 4$.

(III) $F$ is neither indecomposable nor the union of two indecomposable continua. In this event $F$ is not irreducible between $a$ and $b$ and we may have either Case 2 or Case 3 of $\S 4$.

6. Theorem. Let $H$ and $K$ be bounded continua, $H \cdot K \neq 0$ and not connected, $F=H+K$, and $H^{*}=F-K$ and $K^{*}=F-H$ be connected. Let $R$ be a component of $Z-F$, let $a$ and $b$ be points on different components of $H \cdot K$, and let $C$ be $a$ simple arc joining $a$ and $b$ and contained in $R+a+b$. Let $F_{1}$ and $F_{2}$ be the frontiers of the components of $\cdot R-R \cdot C$. Then $F_{1}=C+h$ and $F_{2}=C+k$, where $h$ and $k$ are sub-continua of $H$ and $K$, respectively, joining $a$ and $b$.

† Tôhoku Mathematical Journal, vol. 12, pp. 60-62.

‡ See W.A. Wilson, loc. cit. II, p. 86. 
Let $R_{1}$ and $R_{2}$ be the components of $R-R \cdot C$. and let $S_{1}$ and $S_{2}$ be the components of $Z-(C+H)$ whose frontiers contain $C$. Let $S_{2}$ be the one which contains the connected set $K^{*}$. Since $H \cdot S_{1}=0,\left(H+K^{*}\right) \cdot S_{1}=F \cdot S_{1}=0$ and $S_{1} \subset R$. If $S_{1} \cdot R_{1} \neq 0, S_{1} \cdot R_{2}=0$, since $C \cdot S_{1}=0$; and vice versa. Let then $S_{1} \subset R_{1}$. But then $S_{1}=R_{1}$, since $R_{1} \cdot(C+H)=0$.

Now let $m$ be a point of $R_{1}$ and $n$ one of $R_{2}$. Let $F_{1}$ be the frontier of $R_{1}$ and $h=H \cdot F_{1}$; thèn $F_{1}=C+h$. Either $h$ contains a continuum joining $a$ and $b$, or $h=h_{1}+h_{2}$, where $h_{1} \cdot h_{2}=0, a \subset h_{1}, b \subset h_{2}$, and $h_{1}$ and $h_{2}$ are closed. As neither $h_{1}, h_{2}$, nor $C$ is an $S(m, n)$, but $F_{1}$ is, the latter alternative is impossible by Janiszewski's theorem, loc. cit. As $a$ and $b$ are on different components of $H \cdot K, h \cdot H^{*} \neq 0$.

Now let $h^{\prime}$ be a sub-continuum of $h$ irreducible between $a$ and $b$. If $h$ is not a continuum, $h=h_{1}+h_{2}$, where $h_{1}$ and $h_{2}$ are closed and $h_{1} \cdot h_{2}=0$. As $h \supset h^{\prime}$, either $h^{\prime} \subset h_{1}$ or $h^{\prime} \subset h_{2}$, say the former. But then $F_{1}=C+h=\left(C+h_{1}\right)+h_{2}$ is not a continuum, for $h_{2} \cdot\left(C+h^{\prime}\right)=0$. This is a contradiction.

In like manner there is a component $T$ of $Z-(C+K)$ which is identical with $R_{1}$ or $R_{2}$, and whose frontier contains $C$ and points of $K^{*}$. As $F_{1} \subset C+H$, $T \neq R_{1}$. Hence $T=R_{2}$ and $F_{2}=C+k$, where $k$ is a sub-continuum of $K$.

COROLLARY. If in addition to the above hypotheses $F$ is the frontier of $R$ and the sum $H+K$ is irreducible $\dagger, F_{1}=C+H$ and $F_{2}=C+K$.

For $F_{1}+F_{2}=F+C$ and hence $F_{1} \supset H^{*}$ and $F_{2} \supset K^{*}$. But $\bar{H}^{*}=H$, since $H+K$ is an irreducible sum. Then $F_{1}=C+H$ and $F_{2}=C+K$.

Remarks. A consequence of the above theorem is that not more than two components of $H \cdot K$ contain points arcwise accessible from $R$. (See also \$12.)

Very simple examples show that the omission of the hypothesis that $H^{*}$ and $K^{*}$ are connected renders the theorem invalid and that the hypothesis that $H+K$ is an irreducible sum cannot be omitted from the corollary.

If no statements are made regarding a partition of $F$ into sub-continua and $a$ and $b$ are any two points on $F$, we can only say that the frontier of each of the regions $R_{1}$ and $R_{2}$ contains $C$ and a sub-continuum of $F$ irreducible between $a$ and $b$. This follows readily from Janiszewski's theorem, loc. cit. The two irreducible continua involved may be identical, the simplest example being when $F$ is a straight line segment. In the special case that $a$ and $b$ lie on the frontier of some component of $Z-F$ other than $R$, we can by virtue of the theorem of $\$ 3$ make more precise statements regarding these irreducible continua in accordance with the classification of $\$ 5$.

$\dagger$ This means that there is no proper sub-continuum $H^{\prime}$ of $H$ (or $K^{\prime}$ of $K$ ) such that $F=H^{\prime}+K$ (or $F=H+K^{\prime}$ ). 
7. The theorem of $\$ 6$ is not applicable unless two components of $H \cdot K$ contain points arcwise accessible from $R$, and examples not having this property are easily constructed. If in such a case we desire to separate $R$ by a continuum $C$ into two domains whose frontiers are $C+H$ and $C+K$, continua more general than the simple arc must be used.

We first note that, if $m$ and $n$ are points of the two domains just referred to, $C$ separates $m$ from $n$ in $R$. If, furthermore, $C$ is irreducible with respect to this property and is not itself an $S(m, n)$, it has been shown elsewhere $\dagger$ that $C$ is an irreducible continuum, which may be indecomposable. It seems desirable, however, in the interests of simplicity, to confine the discussion to the case where $C$ is neither indecomposable nor the union of two indecomposable continua. A further justification of this slight loss in generality is the easily established fact that, if $C$ has the separation properties above mentioned and meets the frontier of $R$ in two closed sets $A$ and $B$ such that $A \cdot B=0$, there is a continuum $C^{\prime}$ joining $A$ to $B$ and separating $m$ from $n$ in $R$ irreducibly, which is decomposable into more than two continua.

To save words a decomposable continuum which is not the union of two indecomposable continua will be called semi-separable. It is easily shown that, if $C$ is semi-separable and irreducible between the closed subsets $\alpha$ and $\beta$, then $C=A+E+B$, where $A, B$, and $E$ are continua, $A \cdot B=0, \alpha \subset A$, $\beta \subset B$, and $E$ is irreducible between $A$ and $B$.

8. Theorem. Let $C$ be a bounded semi-separable continuum irreducible between the subsets $\alpha$ and $\beta$. Let no sub-continuum of $C$ separate a point of $\alpha$ from one of $\beta$. Then $Z-C$ has exactly one principal component.

By $\S 7, C=A+E+B$, where $A, B$, and $E$ are continua irreducible between $\alpha$ and $E, \beta$ and $E$, and $A$ and $B$, respectively. Let $a$ and $b$ be points of $\alpha$ and $\beta$, respectively, and let $\epsilon>0$ be so small that $(E+B) \cdot V_{\epsilon}(a)=0$ and $(A+E)$ - $V_{\epsilon}(b)=0$. Set $\delta_{i}=\epsilon / 2^{i}$ and let $V_{i}$ and $W_{i}$ denote the sets of points whose distances from $a$ and $b$, respectively, are less than $\delta_{i}$.

Set $C_{i}=C\left(Z-V_{i}-W_{i}\right)$. Then $C_{i}$ is bounded and closed and is not an $S(a, b)$, since by hypothesis none of its components is an $S(a, b)$. Hence there is a simple arc from $a$ to $b$ not cutting $C_{i}$. Let $L_{i}$ be a segment of this joining $\bar{V}_{i}$ to $\bar{W}_{i}$. Then $C \cdot L_{i}=0$ and $L_{i}$ lies in a component $R$ of $Z-C$.

Now suppose that for some $j \neq i$, the $\operatorname{arc} L_{j}$ were in a different component $S$ of $Z-C$. Let $x$ and $y$ be points of $L_{i}$ and $L_{j}$, respectively. Then $C$ is an $S(x, y)$. But it is obvious that neither $A+E$ nor $E+B$ is an $S(x, y)$ on account of the choice of $\epsilon$, and $(A+E) \cdot(E+B)=E$, a continuum. This is a

† See Wilson, loc. cit. I, §11. 
contradiction by the footnote on page 554 . Hence $S=R$; i.e., for every $i, L_{i}$ lies in the same component $R$ of $Z-C$. Then $R$ contains points in every $V_{i}$ and every $W_{i}$. As $\delta_{i} \rightarrow 0$, the frontier of $R$ must contain $a$ and $b$. Since $C$ is irreducible between $a$ and $b$, it follows that $C$ is the frontier of $R$.

Obviously this can be true of no other component of $Z-C$.

CoROLlary. Let $C$ satisfy the hypotheses of the above theorem and $C=A+E+B$, where $A, E$, and $B$ are continua and $E$ is irreducible between $A$ and $B$. If $S$ is a secondary component of $Z-C$, its frontier is a part of $A+E$ or of $E+B$.

Let $R$ be the principal component of $Z-C$, let $m$ be a point of $R$, and $n$ one of $S$. If $F$ is the frontier of $S, F$ is an $S(m, n)$. The corollary is obviously true unless $F$ contains points in both $A$ and $B$. Then $E+F$ is an $S(m, n)$. But $(E+F \cdot A)(E+F \cdot B)=E$. Hence either $E+F \cdot A$ or $E+F \cdot B$ is an $S(m, n)$, say the former. Let $y$ be a point of $F \cdot B$ not in $E+A$. Then $(E+A) \cdot V_{\delta}(y)=0$ for some $\delta>0$. But $R \cdot V_{\delta}(y) \neq 0$ and $S \cdot V_{\delta}(y) \neq 0$. Hence there is a continuum joining $m$ and $n$ and not meeting $E+F \cdot A$. Thus we have a contradiction unless $F \cdot B=0$.

Remarks. The condition that no sub-continuum $E$ of $C$ may separate a point of $\alpha$ from one of $\beta$ is a necessary one. For in the contrary case $E$ separates every point of $\alpha$ from $\beta$, as $C$ is irreducible between $\alpha$ and $\beta$, and then there would be no principal component of $Z-C$.

The theorem does not remain valid if the word "semi-separable" is omitted. For in Ex. 1, p. 87 of Wilson, loc. cit. II, is given an irreducible continuum whose complement has no principal component. This continuum is the union of two indecomposable continua, but in other respects satisfies the hypotheses of the theorem.

9. Theorem. Let $F=C+K$, where $C$ and $K$ are bounded continua. Let $C \cdot K$ be the sum of two closed sets without common points and let $C$ be semiseparable and irreducible between them. Then $C$ is a proper part of the frontiers of exactly two components of $Z-F$.

By $\$ 13$ of Wilson, loc. cit. I, there are at least two components of $Z-F$ such that $C$ is a proper part of their frontiers. Now $C-C \cdot K$ is connected and lies in one component $R$ of $Z-K$. Then there are at least two points $m$ and $n$ of $R$ such that $C$ is an irreducible $S(m, n)$ in $R$. On the other hand $C$ is not an $S(m, n)$ by $\S 8$ and hence $\$ 11$ of Wilson, loc. cit. I, is applicable. This gives the theorem, since $C$ is semi-separable and hence not irreducible between each pair of three or more closed sets. 
COROLLARY 1. Let $R$ be a simply connected region whose frontier $F$ is bounded. Let $\alpha$ and $\beta$ be closed subsets of $F$ without common points, let $C$ be a bounded continuum contained in $R+\alpha+\beta$, and let $C$ be semi-separable and irreducible between $\alpha$ and $\beta$. Then $C$ is a proper part of the frontiers of exactly two of the simply connected regions into which it divides $R$.

COROLlaRY 2. Let $R$ be a simply connected region whose frontier $F$ is bounded. Let $\alpha$ and $\beta$ be sub-continua of $F$ without common points, let $C$ be a bounded continuum contained in $R+\alpha+\beta$ and containing $\alpha+\beta$, and let $C$ be semi-separable and irreducible between $\alpha$ and $\beta$. Let $C$ separate no two points of $F-C \cdot F$. Then there are exactly two components $R_{1}$ and $R_{2}$ of $R-R \cdot C$ whose respective frontiers $F_{1}$ and $F_{2}$ contain $C$ and $F_{1}+F_{2}=C+F$.

The corollary is obvious unless there is another component $R_{i}(i>2)$ of $R-R \cdot C$. If so, let $F_{i}$ be its frontier and $H_{i}=C \cdot F_{i}$. Then $H_{i} \neq C$ and $H_{i}$ $=K_{i}+L_{i}$, where $K_{i}$ and $L_{i}$ are closed and $K_{i} \cdot L_{i}=\alpha \cdot L_{i}=\beta \cdot K_{i}=0$. Let $x$ be a point of $R_{1}$ and $y$ one of $R_{i}$. Then $F_{i}$, and hence $H_{i}+\alpha+\beta+F$, is an $S(x, y)$. Since $\left(K_{i}+\alpha+F\right)\left(L_{i}+\beta+F\right)=F$, either $K_{i}+\alpha+F$ or $L_{i}+\beta+F$ is an $S(x, y)$, say the former. Now $F$ is not an $S(x, y)$, but $\left(K_{i}+\alpha\right)(\alpha+F)=\alpha$, a continuum. Thus $K_{i}+\alpha$ is an $S(x, y)$.

This shows that $R_{i}$ is a part of a secondary component of $Z-C$, whereas $R_{1}$ is a part of the principal component. If $F_{i}$ contained points of $F-C \cdot F$, it would follow that $C$ separates $F-C \cdot F$, contrary to the hypothesis. Hence $F-C \cdot F \subset F_{1}+F_{2}$ and consequently $C+F=F_{1}+F_{2}$.

Corollary 3. Let $H$ and $K$ be bounded continua, $H \cdot K \neq 0, F=H+K$, and $H^{*}=F-K$ and $K^{*}=F-H$ be connected. Let $R$ be a component of $Z-F$, let $\alpha$ and $\beta$ be closed subsets of two different components of $H \cdot K$, and let $C$ be a bounded semi-separable continuum contained in $R+\alpha+\beta$ and irreducible between $\alpha$ and $\beta$. Let $R_{1}$ and $R_{2}$ be the components of $R-R \cdot C$ whose frontiers $F_{1}$ and $F_{2}$ contain $C$. Then $F_{1} \subset C+H, F_{2} \subset C+K, F_{1} \cdot H^{*} \neq 0$, and $F_{2} \cdot K^{*} \neq 0$.

This follows by the reasoning of the first two and last paragraphs of $\$ 6$.

COROLlaRY 4. Let $R$ be a simply connected region whose frontier $F$ is the irreducible sum of two bounded continua $H$ and $K$, such that $H^{*}=F-K$ and $K^{*}=F-H$ are connected and $H \cdot K$ is not connected. Let $\alpha$ and $\beta$ be closed subsets of two different components of $H \cdot K$ and let $C$ be a bounded continuum contained in $R+\alpha+\beta$ which is semi-separable and irreducible between $\alpha$ and $\beta$. Then there are two components of $R-R \cdot C$ whose respective frontiers are $C+H$ and $C+K$.

By Corollary 3 there are two components $R_{1}$ and $R_{2}$ whose frontiers $F_{1}$ and $F_{2}$ are parts of $C+H$ and $C+K$, respectively, and contain $C$. If there is 
any other component $R_{i}$ of $R-R \cdot C$, it can be shown as in Corollary 2 that any point of $R_{i}$ is separated from one of $R_{1}$ by the union of a proper subcontinuum of $C$ and one of the components of $H \cdot K$. As $H^{*}$ is connected and $F_{1} \cdot H^{*} \neq 0$, the frontier $F_{i}$ of $R_{i}$ contains no point of $H^{*}$. Then, if $U$ is the union of the sets $F_{i}$ for $i>2, \bar{U}$ contains no point of $H^{*}$. Hence $H^{*}+K^{*}$ c $F_{1}+F_{2}$. Since $F=H+K$ is an irreducible sum, $\bar{H}^{*}=H$ and $\bar{K}^{*}=K$. Combining these statements gives the desired result.

Remarks. The requirement in Corollary 2 that $C$ must not separate $F-C \cdot F$ is not trivial or superfluous. To see this, let $F$ be the union of a circumference $M$ and a segment $a b$ of a radius, where $a$ lies on $M$ and $b$ within $M$. Let $R$ be the bounded component of $Z-F, \alpha=a$, and $\beta$ be another point on $M$. Let $C$ be the union of a circumference $N$ lying in $R$ and tangent to $N$ at $a$ and a ray in $R$ starting at $\beta$ and approaching $N$ asymptotically. Then $F_{1}+F_{2}=C+M$, but not $C+F$.

In regard to Corollary 4 it will be shown later by a theorem on accessibility that $\alpha$ and $\beta$ cannot lie on more than two components of $H \cdot K$ and be subsets of a continuum $C$ in $R+\alpha+\beta$. This corollary is false unless both $H^{*}$ and $K^{*}$ are connected.

10. The notion of arc-wise accessibility has been generalized $\dagger$ in a form equivalent to the following definition.

If $F$ is a bounded continuum and $R$ is a component of $Z-F$, the subcontinuum $f$ of $F$ is called accessible from $R$ if for each point a of $R$ there is a bounded continuum $C$ such that $C \subset R+f ; C \supset a ; C \cdot f \neq 0 ; C \cdot F \subset f$.

The choice of $a$ is obviously immaterial. It may well happen that no point of $f$ is accessible from $R$, either by a line or a continuum. As an example, let $F$ consist of a circumference $f$, two spirals approaching $f$ asymptotically, and a chord joining the spirals, and let $R$ be one of the principal components of $Z-F$. Then $f$, but no point of $f$, is accessible from $R$. The following is a special case of a theorem announced in the abstract referred to above. $\dagger$

Theorem. Let $F$ be a bounded continuum and $R$ a component of $Z-F$. Let $f$ be a sub-continuum of $F$ accessible from $R$ and $a$ be any point of $R$. Then there is a raycontained in $R$ whose initial point is a and whose improper limiting points lie on $f$.

11. Theorem. Let $H$ and $K$ be bounded continua, $H \cdot K \neq 0, F=H+K$, and $H^{*}=F-K$ and $K^{*}=F-H$ be connected. If $R$ is a component of $Z-F$, there is no continuum $C$ contained in $R+H \cdot K$ which has points on more than two components of $H \cdot K$.

$\dagger$ G. T. Whyburn, $A$ generalized notion of accessibility, Bulletin of the American Mathematical Society, vol. 34, p. 429 (abstract). 
Let us assume that $R$ is bounded; a similar proof holds if $R$ is unbounded. Now suppose the existence of a continuum $C$ contained in $R+H \cdot K$ and having points on three components $\alpha_{1}, \alpha_{2}$, and $\alpha_{3}$ of $H \cdot K$. Then $H \cdot K=\sum_{1}^{3} A_{i}$, where each $A_{i}$ is closed, no two have common points, and $\alpha_{i} \subset A_{i}$.

Let $P$ be a polygon in $R$ whose interior $I$ contains a point $a$ of $C$ and which is so large that no component of $C-C \cdot I$ connects any pair of the sets $\left\{A_{i}\right\}$. For each $i$ let $C_{i}$ be a sub-continuum of $C$ irreducible between $P$ and $A_{i}$ and let $L_{i}$ be a simple arc within $P$ joining $a$ to $C_{i}$; let $L_{i} \cdot L_{j}=a$ if $i \neq j$. Set $D_{i}=L_{i}+C_{i}$.

Now $D_{1}+D_{2}$ is semi-separable and irreducible between $A_{1}$ and $A_{2}$. By $\$ 9$, Corollary $1, D_{1}+D_{2}$ divides $R$ into several domains of which there are two, $R_{1}$ and $R_{2}$, whose frontiers $F_{1}$ and $F_{2}$ contain $D_{1}+D_{2}$. By Corollary 3 of $\$ 9, F_{1} \subset H+D_{1}+D_{2}$ and $F_{2} \subset K+D_{1}+D_{2}$.

As $D_{3}$ is irreducible between $a$ and $A_{3}$ and $D_{3} \cdot\left(D_{1}+D_{2}\right)=a, D_{3}$ lies in $\bar{R}_{1}$ or $\bar{R}_{2}$, say in $\bar{R}_{2}$. Applying the same references again, we see that there are two components $R_{21}$ and $R_{22}$ of $R_{2}-R_{2} \cdot D_{3}$ whose frontiers contain $D_{1}+D_{3}$ and $D_{2}+D_{3}$, respectively, and a part of $K^{*}$. (For, as shown in the demonstration of $\$ 9$, Corollary 3 , the frontier of $R_{21}$ contains a sub-continuum of $D_{1}+K$ joining $a$ and $A_{3}$.) On the other hand there are two components $S_{1}$ and $S_{2}$ of $R-R \cdot\left(D_{1}+D_{3}\right)$ whose frontiers contain $D_{1}+D_{3}$ and parts of $K$ and $H$, respectively. One of these, say $S_{1}$, equals $R_{21}$. Then $S_{2} \supset R_{1}$, since $R_{1}$ borders the arc $L_{1}$. Also $S_{2} \supset R_{22}$, since $R_{22}$ borders the $\operatorname{arc} L_{2}$. This is a contradiction, since $S_{2}$ does not have any frontier points on $K^{*}$, but $R_{22}$ does. Hence the theorem is proved.

12. THEOREM. Let $F$ be the union of two bounded continua $H$ and $K$ such that $H \cdot K$ is not connected and $H^{*}=F-K$ and $K^{*}=F-H$ are connected. Let $R$ be a component of $Z-F$ having frontier points on both $H^{*}$ and $K^{*}$. Then exactly two components of $H \cdot K$ are accessible from $R$.

By a theorem of Knaster and Kuratowski there is a bounded continuum $P$ which is an irreducible $S\left(H^{*}, K^{*}\right)$ and of course contains points of each component of $H \cdot K$.

Let $M=P \cdot R$. If $\bar{M}$ had points on three components $\alpha, \beta, \gamma$ of $H \cdot K$, we would have a contradiction. For let $A, B, C$ be components of $M$ having limiting points on $\alpha, \beta, \gamma$, respectively. Join $A$ to $B$ and $C$ by $\operatorname{arcs} D$ and $E$ within $R$. Then $\bar{A}+\bar{B}+\bar{C}+D+E$ would be a continuum in $R+H \cdot K$ having points on more than two components of $H \cdot K$, contrary to $\S 11$.

Suppose now that $\bar{M}$ had points on only one component $\alpha$ of $H \cdot K$.

$\dagger$ B. Knaster and C. Kuratowski, Sur les ensembles connexes, Fundamenta Mathematicae, vol. 2, p. 236. 
Let $N=P-M$. Then $Q=\bar{M}+\alpha+N$ is an $S\left(H^{*}, K^{*}\right)$. The set $N+\alpha$ is not an $S\left(H^{*}, K^{*}\right)$, since $R$ has frontier points on both $H^{*}$ and $K^{*}$. Since there is at least one other component of $Z-F$ having this property, $\bar{M}+\alpha$ is not an $S\left(H^{*}, K^{*}\right)$. As $(\bar{M}+\alpha) \cdot(\alpha+N)=\alpha$, a continuum, we have a contradiction.

Thus $\bar{M}$ has points on two components $\alpha$ and $\beta$ of $H \cdot K$. If $\bar{M}$ does not contain a continuum irreducible between $\alpha$ and $\beta, \bar{M}=M_{1}+M_{2}$, where $M_{1}$ and $M_{2}$ are closed and $M_{1} \cdot M_{2}=M_{1} \cdot \beta=M_{2} \cdot \alpha=0$. Then $N+\alpha+\beta$ is a continuum, since $P$ is a continuum. As $M_{1}$ and $M_{2}$ contain points in $R$, neither $M_{1}+N+\alpha+\beta$ nor $M_{2}+N+\alpha+\beta$ is an $S\left(H^{*}, K^{*}\right)$ by the argument of the previous paragraph. But $\left(M_{1}+N+\alpha+\beta\right) \cdot\left(M_{2}+N+\alpha+\beta\right)=N+\alpha+\beta$, a contradiction. Hence $\bar{M}$ contains a continuum irreducible between $\alpha$ and $\beta$ and meeting $F-(\alpha+\beta)$ nowhere. This proves the theorem.

Remark. If in the above the components of $H \cdot K$ accessible from $R$ are points, they are arcwise accessible from $R$. This follows at once from $\$ 10$.

13. Various conclusions can be deduced by combining the theorems of $\S \S 9-12$. The most interesting is perhaps the following.

Suppose that $R$ is a simply connected region whose frontier $F$ is a bounded continuum which is the union of two continua $H$ and $K$ such that $H^{*}=F-K$ and $K^{*}=F-H$ are connected. If $H \cdot K$ is not connected, it follows from $\$ \S 10$ and 12 that there are exactly two components $\alpha$ and $\beta$ of $H \cdot K$, each of which is accessible from $R$ by a ray. Then there is a bounded connected set $C^{*}$ which is contained in $R$ and has these properties: $C^{*}$ is the homeomorphic image of a simple arc and its limiting points form two continua of condensation lying on $\alpha$ and $\beta$, respectively. As $C=\bar{C}^{*}$ is a continuum irreducible between $\alpha$ and $\beta$, we may apply $\S 9$, Corollary 3. This, together with the fact that $C-C \cdot F$ does not cut the plane tells us that $C$ divides $R$ into two domains $R_{1}$ and $R_{2}$, whose frontiers $F_{1}$ and $F_{2}$ contain $C$ and parts of $H$ and $K$, respectively. If, furthermore, $F=H+K$ is an irreducible sum, we know by $\S 9$, Corollary 4 , that $F_{1}=C+H$ and $F_{2}=C+K$. Thus we have a very simple generalization of the division of a region bounded by a simple closed curve into two regions by a simple arc, applicable to very general frontiers and involving only the replacement of the simple arc by a continuum essentially. equivalent to it. $\dagger$

† Since the submission of this paper to the editors, there has been published in the Fundamenta Mathematicae, vol. 12, pp. 214-239, an article by C.Kuratowski (Sur la séparation d'ensembles situes sur le plan) containing a series of theorems, one of which (p. 227, Cor. 1) can be used to obtain this result.

YALE UNIVERSITY; New Haven, Conn. 